\title{
Generalized Darcy's Law in Filtration Theory
}

\author{
Yuri P. Rybakov ${ }^{1, \star}$ and Natalya V. Semenova ${ }^{1, \star \star}$ \\ ${ }^{1}$ RUDN University, Moscow, Russian Federation
}

\begin{abstract}
We study the hydrodynamics of flow in a porous medium modeling the grain filling in filters. Using the lattice approximation, we derive the structure of the current in porous media and obtain the transverse diffusion coefficient $D$ which proves to be proportional to the diameter $d$ of the grains as constituents of the medium. We consider the axially-symmetric stationary flow in a cylindrical filter and show that the vertical velocity takes its maximal value at the wall, this effect being known as the "near-wall" one. We analyze the solution to the Euler equation with the modified Darcy force, which depends not only on the velocity but also on the gradient of the pressure included in the Darcy coefficient. Finally, within the scope of the perturbation method, we derive the main filtration equation and discuss the influence of modifying the Darcy's law on the efficiency of the filtration process.
\end{abstract}

\section{Introduction. Lattice approximation for the flow in porous media}

The main difficulty in the filtration process seems to be the so-called "near-wall" effect, that is an anomalously large value of the flow velocity near the wall due to larger values of the gaps between the wall and the grains, the effectiveness of the filtration being decreasing [1]. In order to take this effect into account, let us first consider the discrete variant of the mass conservation equation and number the lattice vertexes by the indexes $i, j$ (transverse to the flow ) and $k$ (along the flow), the corresponding Cartesian coordinates being $x, y$ and $z$, respectively. Let us denote the local vertical stream of the fluid with the unit density by $G_{i j k}=S_{0} u_{i j k}$, where $u_{i j k}$ is the velocity of the flow and $S_{0}$ is the area of the gap between the grains. Therefore, the local mass conservation law reads

$$
G_{i j k}=r G_{i j k-1}+p\left(G_{i-1 j k-1}+G_{i+1 j k-1}\right)+q\left(G_{i j-1 k-1}+G_{i j+1 k-1}\right),
$$

where the branching coefficients $r, p, q$ are introduced and it is supposed that $p=q$ due to the $x, y$ symmetry of the flow. Inserting (1) into the mass conservation equation

$$
\sum_{i j} G_{i j k}=\sum_{i j} G_{i j k-1}
$$

one immediately gets the following constraint on the branching coefficients: $r+4 p=1$. Identifying now the lattice spacing with the diameter $d$ of the grain, it can be proved through (1), (2) that in the

${ }^{\star}$ e-mail: rybakov_yup@pfur.ru

${ }^{\star}$ e-mail: dobroe_slovo@inbox.ru 
continuous limit the following differential equation holds:

$$
\partial_{z}\left(r u_{z}\right)=d \partial_{x}\left(p \partial_{x} u_{z}\right)+d \partial_{y}\left(p \partial_{y} u_{z}\right) .
$$

One can rewrite the equation (3) in the form of the stationary conservation law: $\operatorname{div} \vec{j}=0$, where the components of the current $\vec{j}$ in cylindrical coordinates $\rho, z$ read: $j_{\rho}=u_{\rho}-D \partial_{\rho} u_{z}, j_{z}=r u_{z}$, and the transverse diffusion coefficient is introduced: $D=p d$. It is worth-while to stress that the effect of the transverse diffusion in porous media is widely discussed in literature [2, 3].

\section{2 “Near-wall” effect and generalized Darcy's law}

The well-known Darcy's law of filtration was found empirically in 1856 by the French engineer H. Darcy [4] who specialized in constructing fountains. According to this law, the velocity of the fluid flow in a porous medium appears to be proportional to the pressure gradient and depends on the structure of the grains as constituent elements of the medium. Later various modifications of the Darcy law were proposed, with the correction terms containing some degrees of the velocity, which was considered as a formal small parameter [5]. As an alternative to such an activity, we suggest a dynamical approach based on the structure of the fundamental Euler equation, which contains the velocity $\vec{u}$, the pressure $P$, the gravity acceleration $\vec{g}$ and the Darcy force:

$$
(\vec{u} \nabla) \vec{u}+\nabla P=\vec{f}=\vec{g}-k_{D} \vec{u} .
$$

We suggest a generalization of the Darcy law by including in $k_{D}$ the natural invariant $I=(\vec{u} \nabla) P$ in the simplest linear form: $k_{D}=k_{0}+k^{*} I$. We intend to analyze the dependence of the filtration process on the coefficient $k^{*}$. First of all, we write down the mass balance equation and the Euler equation (4) in cylindrical coordinates, using the denotations $u_{\rho}=w, u_{z}=u$ :

$$
\begin{gathered}
\rho r \partial_{z} u+\partial_{\rho}\left[\rho\left(w-D \partial_{\rho} u\right)\right]=0, \\
\left(w \partial_{\rho}+u \partial_{z}\right) u+\partial_{z} P=g-\left(k_{0}+k^{*} I\right) u, \\
\left(w \partial_{\rho}+u \partial_{z}\right) w+\partial_{\rho} P=-\left(k_{0}+k^{*} I\right) w,
\end{gathered}
$$

where the invariant $I$ reads $I=\left(w \partial_{\rho}+u \partial_{z}\right) P$. Multiplying the equations (6) and (7) by $u$ and $w$ respectively and adding the results, one finds for the invariant $I$ the expression:

$$
I=\left[1+k^{*}\left(u^{2}+w^{2}\right)\right]^{-1}\left[g u-k_{0} u^{2}-\frac{1}{2}\left(w \partial_{\rho}+u \partial_{z}\right)\left(u^{2}+w^{2}\right)\right] .
$$

Using (8), one can eliminate the pressure $P$ between the equations (6) and (7), the resulting equation for $u$ and $w$ being:

$$
\partial_{\rho}\left[\left(w \partial_{\rho}+u \partial_{z}\right) u+\left(k_{0}+k^{*} I\right) u\right]=\partial_{z}\left[\left(w \partial_{\rho}+u \partial_{z}\right) w+\left(k_{0}+k^{*} I\right) w\right] .
$$

We define the stream function $\Psi$ through the substitution: $\rho r u=-\partial_{\rho} \Psi, \rho\left(w-D \partial_{\rho} u\right)=\partial_{z} \Psi$, the equation (5) being satisfied identically. We now separate the constant part $u_{0}$ of the velocity, with the stream function $\psi_{0}=\psi_{0}(\rho)$. To explain the "near-wall" effect, one can use the perturbation method by putting $\Psi=\psi_{0}+\psi, \quad u=u_{0}+\tilde{u}$, where it is supposed that $\psi \ll \psi_{0}, \tilde{u} \ll u_{0}, w \ll u_{0}$. Within the perturbation strategy, one can simplify the invariant $I$ by putting $I=I_{0}+\tilde{I}$, where

$$
I_{0}=\left(1+k^{*} u_{0}^{2}\right)^{-1}\left(g u_{0}-k_{0} u_{0}^{2}\right), \quad \tilde{I}=\left(1+k^{*} u_{0}^{2}\right)^{-1}\left[\left(g-2 k_{0} u_{0}\right) \tilde{u}-u_{0}^{2} \partial_{z} \tilde{u}\right],
$$


and the smallness of the parameter $\epsilon=k^{*} u_{0}^{2} \ll 1$ is taken into account. Inserting (10) into (9), one can represent the latter equation in the form:

$u_{0}\left(\partial_{\rho z}^{2} \tilde{u}+k^{*} \partial_{\rho} \tilde{I}\right)+\left(k_{0}+k^{*} I_{0}\right) \partial_{\rho} \tilde{u}=u_{0} \partial_{z}^{2} w+\left(k_{0}+k^{*} I_{0}\right) \partial_{z} w, \quad \tilde{u}=-\frac{1}{\rho r} \partial_{\rho} \psi, \quad w=\frac{1}{\rho} \partial_{z} \psi+D \partial_{\rho} \tilde{u}$.

Taking into account that $D(\rho)=p(\rho) d, r(\rho)=1-4 p(\rho)$, one can solve (11) by separation of variables: $\psi=R(\rho) Z(z), Z=\exp (-\lambda z), \lambda=$ const. The resulting equation for $R(\rho)$ is:

$$
\begin{aligned}
\left(\frac{1}{\rho r} R^{\prime}\right)^{\prime}\left[\lambda u_{0}-k_{0}-k^{*} u_{0}(2 g-3\right. & \left.\left.k_{0} u_{0}+\lambda u_{0}^{2}\right)\right]+ \\
& +\lambda\left[\frac{\lambda}{\rho} R+D\left(\frac{1}{\rho r} R^{\prime}\right)^{\prime}\right]\left[\lambda u_{0}-k_{0}-k^{*} u_{0}\left(g-k_{0} u_{0}\right)\right]=0 .
\end{aligned}
$$

In view of the fact that the branching coefficient $p(\rho)$ vanishes at the wall of the filter, i.e. at $\rho=a$, one gets $p(a)=0$ and derives from the boundary condition $w(0)=w(a)=0$ that for the function $R(\rho)$ : $R(0)=R(a)=0$.

We are now in a position to remark that in the model in question, beside the parameter $\epsilon$, another small parameter can be introduced: $\mu=d / a \ll 1$. Therefore, we can use the perturbation decomposition:

$$
R=R_{0}+\epsilon R_{1}+\mu R_{2}+\cdots, \quad \lambda=\lambda_{0}+\epsilon \lambda_{1}+\mu \lambda_{2}+\cdots,
$$

which permits us to deduce from (12) the following equation for $R_{0}$ :

$$
\left(\frac{1}{\rho r} R_{0}^{\prime}\right)^{\prime}+\frac{\lambda_{0}^{2}}{\rho} R_{0}=0
$$

It is reasonable to consider first the domain $0 \leq \rho<a-d$, for which $r(\rho)=r_{0}=$ const. Using the substitution: $R_{0}=\rho K_{-}(x), x=\rho \lambda_{0} \sqrt{r_{0}}$, one reduces (13) to the Bessel equation, with the solution $K_{-}(x)=A J_{1}(x), A=$ const. On the other hand, in the very small domain $a \geq \rho>a-d$, where $R_{0}(a)=0$, one can put $r \approx 1, R_{0}=\rho K_{+}\left(\rho \lambda_{0}\right)$ and use the approximation: $K_{+} \approx B \lambda_{0}(\rho-a), B=$ const. Matching the functions $K_{-}$and $K_{+}$, together with their first derivatives, at the point $\rho=a-d$, one gets the following algebraic system:

$$
A J_{1}\left[(a-d) \lambda_{0} \sqrt{r_{0}}\right]=-B \lambda_{0} d ; \quad A J_{1}^{\prime}\left[(a-d) \lambda_{0} \sqrt{r_{0}}\right] \sqrt{r_{0}}=B .
$$

As can be easily seen, the system (14) is equivalent to the condition:

$$
J_{1}\left[(a-d) \lambda_{0} \sqrt{r_{0}}\right]=-J_{1}^{\prime}\left[(a-d) \lambda_{0} \sqrt{r_{0}}\right] \sqrt{r_{0}} \lambda_{0} d .
$$

Taking into account that $a \gg d$, one concludes that the condition (15) yields the following equation for $\lambda_{0}$ :

$$
J_{1}\left(a \lambda_{0} \sqrt{r_{0}}\right)=0 .
$$

Denoting the $n$-th nontrivial zero of the Bessel function $J_{1}(x)$ as $x_{n}$, one can represent the general solution to the equation (11) as follows:

$$
\psi=\sum_{n=1}^{\infty} A_{n} \rho J_{1}\left(\rho \lambda_{0}^{n} \sqrt{r_{0}}\right) \exp \left(-\lambda_{0}^{n} z\right),
$$


where $\lambda_{0}^{n}=x_{n} / a \sqrt{r_{0}}$. In particular, for the first mode one finds the root $x_{1} \approx 3.8317$. As for the amplitudes $A_{n}$ in (17), they can be obtained from the initial velocity profile (at $z=0$ ). It is worthwhile to underline that the solution (17) permits one to explain the aforementioned "near-wall" effect. In fact, if one takes into account that the main contribution to the velocity profile is given by the first mode, one easily finds from the well-known identity for the Bessel functions: $x J_{0}(x)=J_{1}(x)+x J_{1}^{\prime}(x)$ that the vertical velocity near the wall of the filter proves to be proportional to $J_{0}\left(x_{1}\right)$, i. e. it takes its maximal value at the wall. Let us now estimate the constant part $u_{0}$ of the velocity. To this aim, we consider the equation (6) at $\rho=0$ :

$$
u \partial_{z} u-g+k_{0} u+\partial_{z} P\left(1+k^{*} u^{2}\right)=0
$$

and integrate its left-hand side over $z$ in the interval $z \in[0, \ell]$, where $\ell$ stands for the height of the filter. The result of integration can be approximately represented as follows:

$$
\triangle P\left(1+k^{*} u_{0}^{2}\right)+\left(g-k_{0} u_{0}\right) \ell=0
$$

where $\triangle P=P(z=0)-P(z=\ell)$, and the smallness of the parameters $\epsilon, \mu$ is taken into account.

\section{Conclusion}

We analyzed for the first time the origin of the "near-wall" effect in the filtration practice and showed its connection with the transverse diffusion coefficient vanishing at the wall of the filter. The other problem concerned the generalization of the Darcy's law, which was motivated by the idea that the Darcy coefficient $k_{D}$ should have a dynamical origin. To this end, we inserted in $k_{D}$ the natural dynamical invariant $I$ in the simplest linear form $k_{D}=k_{0}+k^{*} I$ and showed that the main filtration relation (19) proved to be modified via deforming the pressure: $P \rightarrow P\left(1+k^{*} u_{0}^{2}\right)$. As a result, the filtration velocity $u_{0}$ should be also modified.

\section{Acknowledgement}

This work was financially supported by the Ministry of Education and Science of the Russian Federation (the Agreement number 02.a03.21.0008).

\section{References}

[1] V.N. Lukerchenko, D.N. Maslov, Yu.P. Rybakov, T.M. Shabalina, G.N. Shikin, and V.A. Yanushkevich, Mathematical Models of Nonlinear Phenomena, Processes and Systems: From Molecular Scale to Planetary Atmosphere. Chapter 14. (Editors: A.B. Nadykto, L.A. Uvarova; Nova Science Publishers, Hauppauge, New York, 2013) Pp. 217-224

[2] K. Vafai, Handbook of Porous Media (CRC Press, Boca Raton, FL, 2015) 959 pp.

[3] F.A.L. Dullien, Porous Media: Fluid Transport and Pore Structure (Academic Press, San Diego, 2012) $574 \mathrm{pp}$.

[4] A.E. Sheidegger, The Physics of Flow Through Porous Media (MacMillan, New York, 1960) $256 \mathrm{pp}$.

[5] P.Ya. Polubarinova - Kochina, Theory of Ground Water Movement (Princeton University Press, Princeton, 1960) $664 \mathrm{pp}$. 